\title{
Temporal and spatial variation in the morphology of the brown macroalga Hormosira banksii (Fucales, Phaeophyta)
}

\author{
Catriona M.O. Macinnis-Ng ${ }^{\star}$, David A. \\ Morrison a and Peter J. Ralph \\ Institute for Water and Environmental Resource \\ Management, The University of Technology, Sydney, \\ Westbourne Street, Gore Hill 2065, Australia, e-mail: \\ Cate.Macinnis@uts.edu.au \\ *Corresponding author
}

\begin{abstract}
Hormosira banksii is a morphologically variable macroalgal species from southeastern and southern Australia, which has been previously categorised into ecoforms according to habitat. This study is by far the largest quantitative evaluation of morphological variation in $\mathrm{H}$. banksii, covering 74 sites from South Australia, Victoria, New South Wales and Tasmania. Morphological features from 505 samples were analysed using principal components analysis, with the patterns identified being statistically assessed with a Monte Carlo permutation test. There was considerable morphological variation between samples taken at several marine (but not estuarine) sites in both 1994 and 1999. However, this variation was not consistent across either morphological features or populations, and presumably represents random fluctuations. Analysis of the entire dataset demonstrated a significant difference between samples growing in marine and estuarine habitats. Further assessment of variation within these two groups revealed some significantly different populations based on geographical locations but not habitat variation. While this study presents strong evidence for two distinct taxa within $H$. banksii (marine versus estuarine populations), the taxonomic status of this species should not be altered until genetic studies have been conducted.
\end{abstract}

Keywords: Australia; Hormosira banksii; morphological variation.

\section{Introduction}

Hormosira banksii (Turner) Decaisne is a brown macroalga common to southeastern and southern Australia. The thallus consists of branched chains of water-filled vesicles (or receptacles), spherical to elongate in shape (Womersley 1987), but the morphology is very variable. Existing only in Australia, New Zealand and nearby islands (Womersley 1967), H. banksii is the only species

\footnotetext{
- Current address: Department of Parasitology (SWEPAR), National Veterinary Institute and Swedish University of Agricultural Sciences, 75189 Uppsala, Sweden
}

of the family Hormosiraceae, typified by a group of four three-sided apical cells at the growing point (Nizamuddin 1961, Clayton 1984). Of the order Fucales, H. banksii is the only intertidal species in the Southern Hemisphere (Womersley 1981).

On mainland Australia, Hormosira banksii is found from King George's Sound in Western Australia (Womersley 1967), around the southern coast to north of Coffs Harbour in New South Wales (Millar and Kraft 1994). It is also present around the coast of Tasmania, both islands of New Zealand and Lord Howe and Norfolk Islands (Womersley 1967). Throughout this range, $H$. banksii is found in three different habitats. Rock pools, rock platforms (both on open coasts) and estuarine habitats each provide different environmental conditions. Estuarine thalli are found growing on rocks and other debris on tidal flats, as well as occurring as a free-living form trapped among mangrove pneumatophores (King 1981. King and Wheeler 1985).

Estuarine thalli are protected from wave and wind exposure, but can experience changes in salinity with low tide, as well as desiccation and temperature changes. Algae on rock platforms tend to be exposed to strong winds and desiccation. Light and temperature conditions can be highly variable on rock platforms, as emersion at low tide leaves the algae exposed. Rock pools on the lower platform can be relatively stable habitats, in contrast to pools on the upper platform where salinity and temperature increase at low tide due to the evaporation of water and exposure to the sun. These environmental conditions (particularly wave action and degree of desiccation) influence the physiology of $\mathrm{H}$. banksii and have been found to correlate with morphological variation (Osborn 1948, Bergquist 1959, Womersley 1967, Ralph et al. 1998).

Morphological variation influenced by characteristic seasonal or habitat features is common among the brown algae (for example, Rice and Chapman 1985, Bhattacharya and Druehl 1989, Cheshire and Hallam 1989, Cheshire and Conran 1995, Miller et al. 2000, Serisawa et al. 2003), making differentiation between taxa difficult (Russell 1978). Cross-fertilisation experiments involving several fucoid species (Burrows and Lodge 1951, Clarke and Womersley 1981) show that they are not necessarily reproductively isolated. Some phenotypic traits are plastic while others are stable (Russell 1986, Serisawa et al. 2003), so the value of morphological studies may be limited, yet genetic studies can be highly labour-intensive and generally reveal that algae (and members of Phaeophyta in particular) are extremely conservative in comparison to other plants (Bhattacharya and Druehl 1989, Neefus et al. 1993).

Morphological studies of Hormosira banksii from different microhabitats have been used previously in an attempt to determine the nature of variation in the spe- 


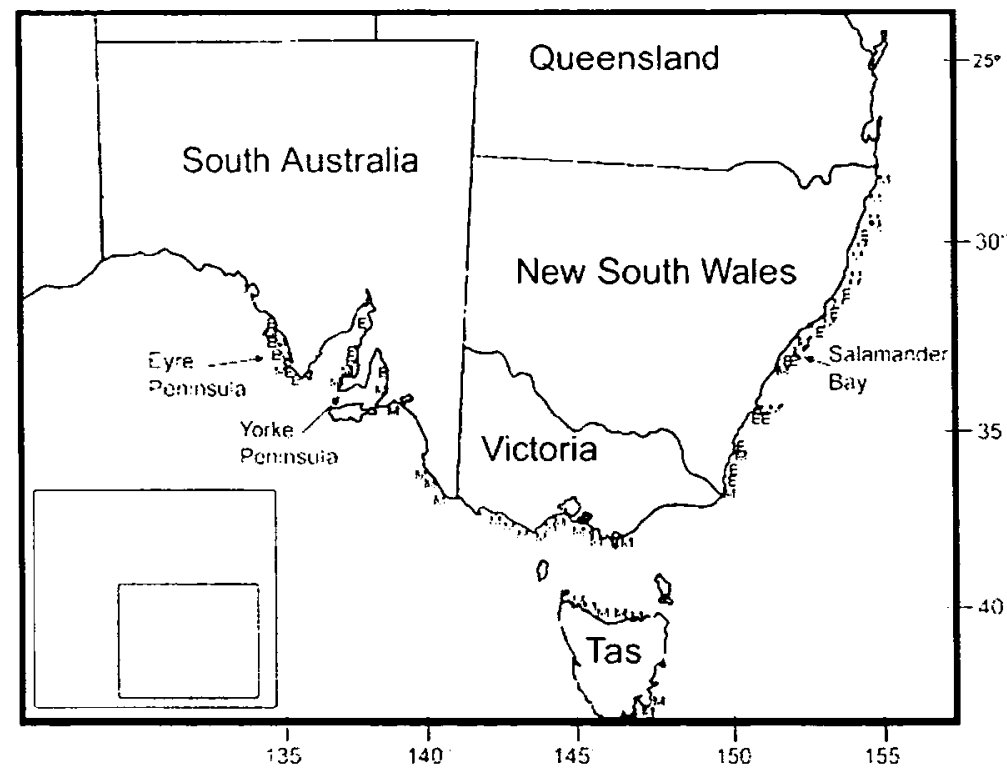

Figure 1 Hormosira banksii: distribution of sample sites in southeastern Australia

Marine sites are marked ' $M$ ' and estuarine sites are marked ' $E$ '. ' denotes sites sampled in 1994 and 1999 for the temporal variation assessment.

cies, but the results remain inconciusive, mainly due to the limited geographical distributions of the studies. A study of variation in $H$. banksii throughout New Zealand was reported by Bergquist (1959), while the most extensive study of Australian forms covered only $300 \mathrm{~km}$ of the New South Wales coast (Ralph et al. 1998). Such a limited study of the distribution of $H$. banksii within Australia may fail to detect morphological differences due to geographical location (Ralph et al. 1998). Furthermore. the potential for temporal variation in morphology has not yet been investigated at all.

The current study determines whether morphologically distinct groups exist within the species Hormosira banksii across its range in Australia. We also looked at associations between environmental conditions and morphological variation. The principal aim was to determine whether morphology of $H$. banksii varies consistently with the habitat (marine platforms and pools and estuarine areas), microhabitat within habitats (tidal height or location in a rock pool) and geographical distribution. We also assessed temporal variation in $H$. banksii by comparing data collected in 1994 (reported in Ralph et al. 1998) with data collected at the same sites in 1999. We suggest that low dispersal of gametes due to release at low tide (a common trait in Phaeophyceae; Williams and DeFiori 1996, Coyer et al. 1997. Brawley et al. 1999, Beligrove et al. 2004) has led to morphologically discrete populations of $H$. banksii which are adapted to different habitats.

\section{Materials and methods}

Field trips were conducted along the south-eastern coast of mainland Australia. from the Queensland border to the Eyre Peninsula and to Tasmania to collect samples from three habitats (rock platforms and pools and estuarine areas, including mangroves) between January 1998 and January 2001 at 74 sites (Figure 1). Sites were divided into microhabitats according to their tidal level for rock platforms (habitat 1) and estuarine areas (habitat 2: upper, middle and lower tidal) and according to their position in the pool (habitat 3: surface, bottom, edge) as described by Ralph et al. (1998).

At each site, samples (one thallus arising from a single holdfast) were removed at the holdfast, placed in labelled plastic bags and kept on ice. Environmental conditions such as weather, substratum, degree of exposure, aspect and other algae present in the community were recorded. Length and diameter of ten healthy vesicles from each of the three thalli collected at each microhabitat (generally nine samples per site) were measured with digital vernier calipers (Mitutoyo, CD-6"C, Kawasaki, Japan) to the nearest $0.1 \mathrm{~mm}$. Vesicles measured were mature. nonbranching and of representative size in the middle of the branch. The shape of each vesicle was recorded as cylinder, ovoid or cup/cone shaped. The length, diameter and shape measurements allowed the later estimation of the volume, surface area, surface area:volume ratio and length:diameter ratio. The number of vesicles from the terminal (growing) tip to the first branch was counted as a representation of the branching pattern. In the rare instance where there was no branch, we counted the number of vesicles from the holdfast. The mean length, diameter, branching pattern, surface area, volume, surface area:volume ratio and length:diameter ratio were then calculated for each plant.

Principal components analysis (PCA; ter Braak 1988) was used to analyse the patterns of morphological variation among the 505 individual thalli measured in 1998-2001. This multivariate pattern-analysis technique derives a small number of linear functions (one less than the number of variables) that attempt to summarise as much as possible of the morphological similarity based on all of the attributes simultaneously. The attributes were each separately range standardised in order to 
make them directly comparable. For display, the ordination summary provided by the first two functions was examined, along with an indication of which of the morphological attributes showed the most variation among the individuals. Alternative multivariate analyses were investigated (including other metric analyses such as correspondence analysis, and non-metric analyses such as multidimensional scaling and analysis of similarities), and these all produced qualitatively the same results; and so only the PCA results are presented here, as being the most informative.

A sequential series of several analyses was used, each subsequent analysis analysing in more detail those patterns identified in previous more-inclusive analyses. Sequential analyses were based on major physiological distinctions (marine versus estuarine samples) and geographical location. The 'southern coastline' included samples from South Australia, Victoria and Tasmania while the 'eastern coastline' was the New South Wales samples [loosely following Knox's (1963) algal biogeographic provinces]. The "northern part of the New South Wales coastline" included all samples north of Sydney and the "southern part of the New South Wales" coastline included all samples south of Sydney.

To assess statistically the patterns identified, a Monte Carlo permutation test was used, based on a redundancy analysis of the data (RDA; ter Braak 1988). Redundancy analysis is a constrained ordination technique based on PCA, which assesses the degree to which two data sets show covariation. For our analyses, the first data set was the morphological data and the second data set specified the pattern being tested. In all cases, 2000 permutations were used.

For the assessment of temporal variation in the morphological attributes at the same sites. seven data variables were used: vesicle length $(\mathrm{mm})$. vesicle diameter $(\mathrm{mm})$. wall thickness $(\mathrm{mm})$, vesicle volume $\left(\mathrm{mm}^{3}\right)$. cavity volume $\left(\mathrm{mm}^{3}\right)$. vesicle surface area $\left(\mathrm{mm}^{\bar{c}}\right)$. and surface area:volume ratio (In transformed). Data were collected at the following sites: Carama Inlet $\left(35^{\circ} 00^{\prime}\right.$ 's, $150^{\circ} 45^{\circ} \mathrm{E}$ ) and Towra Point (mangrove habitat, $34^{\circ} 02^{\prime} S$, $\left.151^{\circ} 15^{\circ} \mathrm{E}\right)$. Plantation Point $\left(35^{\circ} 05^{\prime} \mathrm{S}, 150^{\circ} 40^{\prime} \mathrm{E}\right)$ and Swansea Heads (rock platform habitat, $33^{\circ} 06^{\prime} S$, $151^{\circ} 40^{\prime} \mathrm{E}$ ), and Plantation Point and Swansea (rock pool habitat). All of these sites were sampled in 1999 as part of the larger data set. In 1994, there were six thalli sampled per microhabitat for the mangrove and pool sites but ten thalli per microhabitat at the platform sites (see Ralph et al. 1998): in 1999, there were three thalli per microhabitat from all sites.

Each variable was analysed separately for each habitat with a three-factor orthogonal analysis of variance. These factors were:

- Site: random factor, two sites per habitat

- Microhabitat: fixed factor, three microhabitats per habitat; platform: exposed, lower eulittoral, sublittoral; pool: surface, edge. bottom; mangrove: high-tidal, mid-tidal, low-tidal

- Time: random factor, two times: 1994, 1999

The appropriate mean squares for testing each factor and interaction in this model were determined using Zar
(1984). Using this model, it is not possible to test the microhabitat factor alone, as there is no appropriate denominator for the F-ratio, unless one calculates a pseudo F-value. Homogeneity of variances was assessed using Levene's test. If the variances were not equal then the data were transformed to natural logarithms. If the In-transformed data were still heterogeneous, then outlying values were deleted. Analyses were performed using Minitab (version 12).

\section{Results}

For the analyses of variance of the temporal variation in the platform and pool habitats (Table 1), either the threefactor interaction between time, site and microhabitat is statistically significant for all of the morphological variables or the two-factor interaction between time and site is significant. This indicates that some of the morphological forms had statistically different measurements at the two times, under at least some combination of the levels of the other two factors (site and microhabitat) Thus, we may reject the null hypothesis that time has no effect at all. However, the differences between the two times for the mean values of the different attributes of each form at each site (Table 2) show that there is no consistent pattern to this variation through time for any of the attributes. For each morphological form, some sites had values that increased and some sites had values that decreased or remained unchanged; and this is true for all of the morphological characteristics. The variation is thus apparently arbitrary.

On the other hand, only two of the hypothesis tests involving the factor time are significant (as a main effect, with no significant interactions) for the data from the mangrove habitat (Table 1), and these are likely to be false positives resulting from the large number of tests performed $(42 \times 0.05=2.1$ expected false positives). Thus, we conclude that temporal variation is not an important influence on the samples from this estuarine habitat.

Therefore, our pilot study demonstrates that Hormosira banksii vesicles can potentially vary in size and shape through time, at least under some circumstances in the marine habitats. However, this can be interpreted as random genetic or environmental variation (based on the reasonable assumption that we have successfully excluded the potential confounding variables), as there is no apparent consistent pattern of variation. Therefore, for our purposes, measurements taken at different times can be treated as samples of that variation. It is thus not invalid to compare data sampled over several years at several locations in our main study.

The first two functions from the principal components analysis of the entire 1998-2001 data set are shown in Figure $2 \mathrm{~A}$, these axes accounting for $73.9 \%$ of the total variation in the morphological attributes. The morphological similarities among the individuals are indicated by the spatial relationship of the points on the ordination diagram: points near to each other show more similarity among themselves (based on the morphological characteristics) than they do to points further away. The variation in the morphological attributes is shown by the length and direction of the arrows: longer arrows indicate 
Table 1 Hormosira banksii: results of the 21 analyses of variance of the temporal variation in morphology, with each morphological variable tested separately for each habitat.

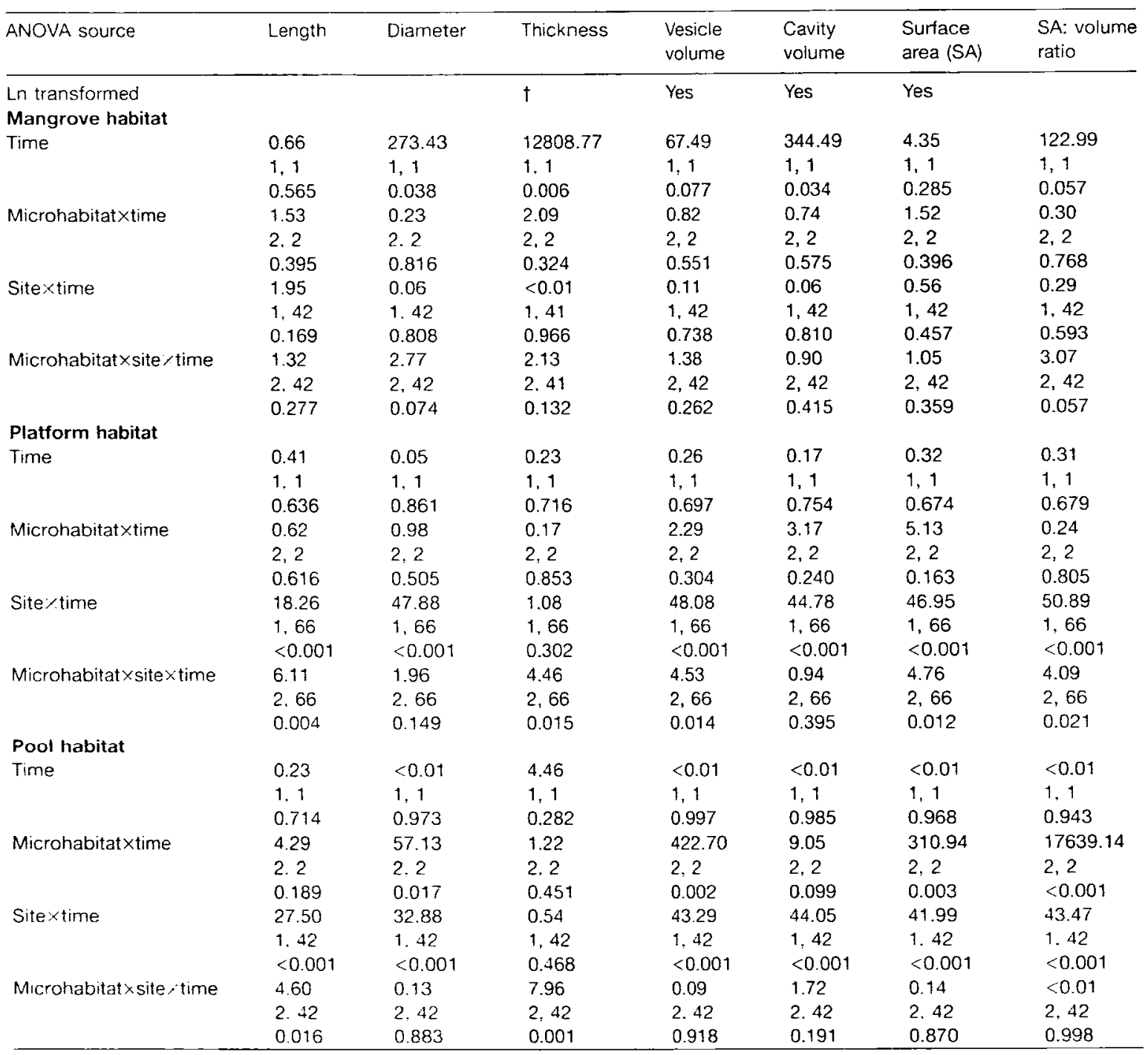

Only those ANOVA sources involving the time factor are shown. For each result, we give, in order, the F-value. the degrees of freedom (hypothesis. error) and the $p$ value. $f$ One outlier deleted from the mangrove sample.

attributes with more morphological variation, and orthogonal arrows indicate unrelated attributes.

This ordination analysis shows that the individuals from the estuarine and the marine sites are (with a few exceptions, see below) morphologically different from each other, these two groups being separated along axis 1 of the ordination diagram (compare Figure $2 \mathrm{~B}$ and $\mathrm{C}$ ). The permutation test indicates that this separation is statistically significant $(F=209.83, p<0.0005)$. The morphological differentiation is related mainly to the size attributes (i.e., their arrows all point roughly parallel to axis 1 ) rather than to the shape or branching attributes, with the estuarine thalli having larger vesicles than the marine thalli. All of the morphological attributes show similar amounts of variation among the individuals (i.e., the arrows are all approximately equal in length), with the exception of the branching attribute.
In order to analyse the morphological variation among these two groups of thalli in more detail, and to identify those relatively few individuals that show apparent morphological similarity between the groups, the estuarine thalli and the marine thalli were analysed separately using PCA.

The first two functions from the principal components analysis of the estuarine data set are shown in Figure 3A, these axes accounting for $93.8 \%$ of the total variation in the morphological attributes. All of the morphological attributes show similar amounts of variation among the individuals, with the exception of the shape attribute (all of the estuarine individuals are roughly spherical), although they are not highly correlated (i.e., they do not all point in the same direction).

The morphological variation among the individuals appears to differentiate into a number of ecological and 
Tabie 2 Hormosira banksir: difference in morphological measurements between the two sampling times (1994 sample minus 1999 sample) for each of the sites and forms.

\begin{tabular}{|c|c|c|c|c|c|c|c|c|c|}
\hline Habitat & Microhabitat & Site & $\begin{array}{l}\text { Vesicle } \\
\text { length } \\
(\mathrm{mm})\end{array}$ & $\begin{array}{l}\text { Vesicle } \\
\text { diameter } \\
(\mathrm{mm})\end{array}$ & $\begin{array}{l}\text { Wall } \\
\text { thickness } \\
(\mathrm{mm})\end{array}$ & $\begin{array}{l}\text { Vesicle } \\
\text { volume } \\
\left(\mathrm{mm}^{3}\right)\end{array}$ & $\begin{array}{l}\text { Cavity } \\
\text { volume } \\
\left(\mathrm{mm}^{3}\right)\end{array}$ & $\begin{array}{l}\text { Surface area } \\
\left(\mathrm{SA} ; \mathrm{mm}^{2}\right)\end{array}$ & $\begin{array}{l}S A: \\
\text { volume } \\
\text { ratio }\end{array}$ \\
\hline \multirow{3}{*}{ Mangrove } & High & 2 & 1.0 & 0.9 & -0.2 & 259.0 & 247.3 & 75.6 & -0.11 \\
\hline & \multirow[t]{2}{*}{ Mid } & 1 & 1.1 & 2.1 & -0.1 & 463.8 & 342.8 & 133.6 & -0.15 \\
\hline & & 2 & -1.3 & 0.2 & -0.5 & 59.9 & 240.8 & -33.0 & -0.09 \\
\hline \multirow[t]{5}{*}{ Platform } & \multirow[t]{2}{*}{ Exposed } & 1 & -1.8 & -1.8 & -0.2 & -307.0 & -122.5 & -184.6 & 0.14 \\
\hline & & 2 & -0.7 & -0.9 & 0.0 & -30.9 & -26.9 & 6.9 & 0.12 \\
\hline & \multirow[t]{2}{*}{ Eulittoral } & 1 & 1.4 & -0.1 & 0.2 & 82.0 & 3.6 & 74.6 & -0.03 \\
\hline & & 2 & 1.3 & 1.6 & 0.2 & 149.3 & 67.2 & 45.3 & -0.38 \\
\hline & Sublittoral & 1 & 2.6 & 1.2 & 0.0 & 452.4 & 199.4 & 252.8 & -0.22 \\
\hline \multirow{3}{*}{ Pool } & Edge & 2 & -0.8 & 0.3 & -0.3 & -42.9 & 2.3 & -30.0 & 0.09 \\
\hline & \multirow[t]{2}{*}{ Bottom } & 1 & 4.2 & 2.1 & 0.4 & 553.9 & 200.8 & 315.9 & -0.36 \\
\hline & & 2 & 2.3 & 0.8 & 0.1 & 80.4 & 36.7 & 32.8 & -0.31 \\
\hline
\end{tabular}

geographical groups. The individuals from the estuarine areas of the southern (South Australian) coastline cluster towards the bottom of axis 2 (Figure $3 \mathrm{~B}$ ), having a small length:diameter ratio and short branch length, and appear to form two geographical groups, based on the York and Eyre Peninsulas (Figure 1). The individuals from the estuarine areas of the eastern (New South Wales) coastline cluster towards the top of axis 2 (Figure $3 \mathrm{C}$ and D). having almost no overlap on the diagram with the thalli from the same habitat along the southern coastline. Furthermore, these thalli appear to differentiate along axis 2 based on their degree of tidal exposure, with those individuals in the lower tidal zone towards the top of the axis and those from the upper tidal zone further down the axis (with no overlap on the diagram). and the thalli from the mid-tidal zone in between. Finally, the individuals from the mangrove sites cluster towards the middle and bottom of axis 2 (Figure $3 \mathrm{E}$ and F). They show some differentiation along this axis in relation to their degree of tidal exposure, but this is not as clear as it is for the tidalflat thalli. Furthermore, all of the thalli from the Salamander Bay $\left(32^{\circ} 46^{\prime} S, 152^{\circ} 05^{\prime} E\right.$, N.S.W. $)$ site are clearly separated along axis 1, their vesicles having a larger surface area and volume than those of the other mangrovehabitat thalli. Salamander Bay is on the southern shores of Port Stephens but how conditions there may have influenced sample morphology is unclear. The permutation tests indicate that the separation of the three main groups (southern coastline, eastern coastline and mangroves) is statistically significant ( $F=22.35, p<0.0005)$, as is the separation of the two geographical areas (southern coastline and eastern coastline) within the tidal-flat areas of South Australia $(F=17.60, p<0.0005)$ and the three tidal heights within the tidal-flat areas of the eastern coastline $(F=4.50, \quad p=0.0465)$. However, the apparent differentiation of the three tidal heights within the mangrove areas is not statistically significant $(F=0.94$, $\mathrm{p}=0.6105$ )
The first two functions from the principal components analysis of the marine data set are shown in Figure 4A, these axes accounting for $65.8 \%$ of the total variation in the morphological attributes. Most of the morphological attributes show similar amounts of variation among the individuals, with the exception of the branch and length:diameter attributes, and the size attributes are highly correlated with each other. Thus, variation along axis 1 represents variation in vesicle size while variation along axis 2 represents variation in vesicle shape.

The morphological variation among the individuals does not appear to form clear groups, although consistent geographical patterns can be discerned. Axis 1 separates some of the individuals along the Victorian coastline (Figure $4 \mathrm{~B}$, to the right) from the rest of the marine thalli (to the left), these individuals having generally larger vesicles. In fact, it is these individuals that are morphologically similar to the estuarine thalli (see Figure 2). The sites from which these thalli were sampled were less exposed to wave action than were the other sites. Axis 2 forms three concentrations of individuals based on vesicle shape, with ellipsoid (on average) vesicles to the bottom of the axis, cylindrical vesicles in the middle and conical vesicles at the top. The raw data show that individuals from the northern New South Wales (Figure 4C) and the South Australian coastlines (Figure 4E) have predominantly cylindrical vesicles, while the individuals from the southern New South Wales (Figure 4D), Victorian (Figure 4B) and Tasmanian coastlines (Figure 4F) have predominantly ellipsoid vesicles. Conical vesicles are absent from the northern New South Wales coastline (Figure $4 \mathrm{C}$ ) but are common along the southern New South Wales coastline (Figure 4D) as well as occurring in Tasmania (Figure 4F). The permutation tests indicate that the separation of the Victorian thalli along axis 1 is statistically significant ( $F=90.16, p<0.0005)$, as is the separation of the five geographical areas along axis 2 $(F=14.87, p<0.0005)$. 

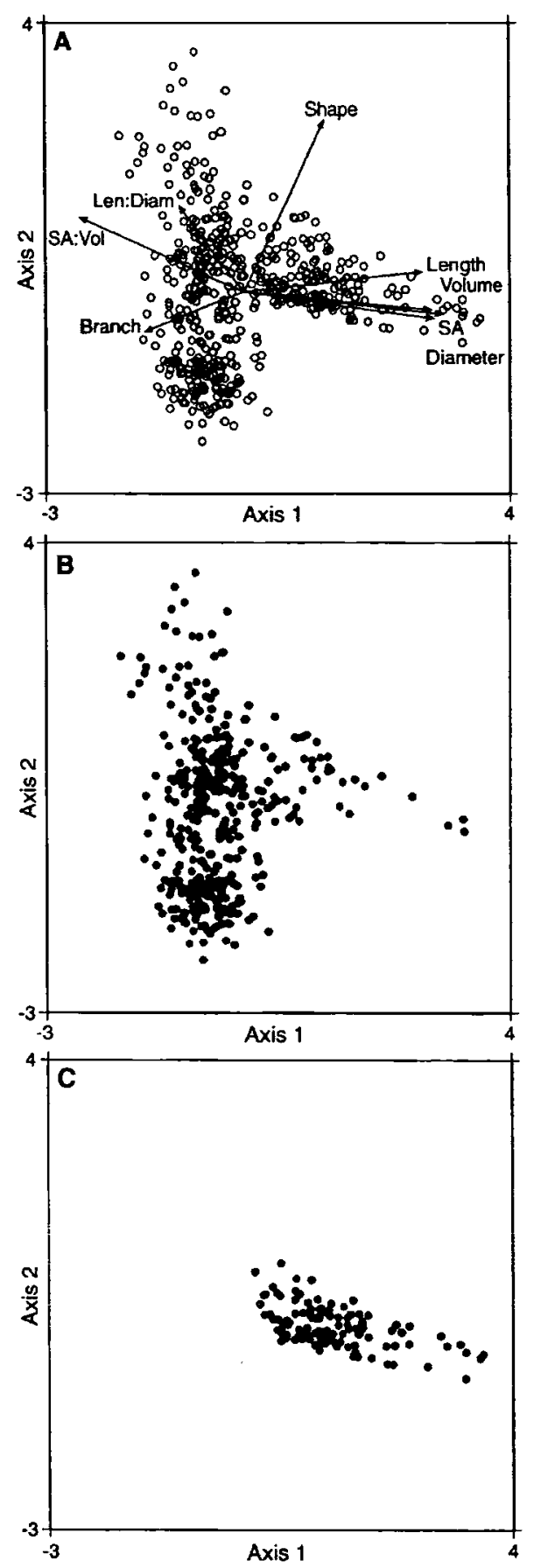

Figure 2 Hormosira banksii: projection of individuals onto axes representing the first two functions of the principal components analysis of the vesicle characteristics.

Showing (A) all 505 individuals from all of the sites, along with the variation in each morphological attribute, $(B)$ only those 380 individuals from the marine sites, and $(C)$ only those 125 individuals from the estuarine sites. Each symbol represents one thallus, and the spatial relationship of the symbols represents their relative morphological similarity based on all of the attributes. Each arrow represents one morphological attribute (Shape: sphere $\rightarrow$ cylinder $\rightarrow$ cone; SA: surface area; Len:Diam: length:diameter ratio; SA:Vol: surface area:volume ratio; Branch: number of branches per chain), the direction of the arrow indicating the direction of maximum variation in that attribute and the length of the arrow indicating the relative amount of morphological change.

\section{Discussion}

This study has successfully assessed a much broader geographical distribution of morphological variation in Hormosira banksii than any previous study. The significant difference found between estuarine and marine samples supports the suggestion by Ralph et al. (1998) that these are two phenotypically independent groups. Beyond the marine/estuarine distinction, the current results reveal a lack of consistently identifiable morphological populations by habitat, despite the findings of Bergquist (1959) and Ralph et al. (1998) in more restricted geographical areas. The current analysis suggests that morphological variation of $H$. banksii within the two main groups was influenced more by geography than by microhabitat type. Yet the variation within the data set showed groupings and was not a multivariate continuous dataset, in contrast to the findings of Osborn (1948), Clarke and Womersley (1981), King (1981) and Womersley (1987) who suggested gradual, continuous changes across habitats and geographical locations.

Despite the influence of geographical distribution on the patterns found, there are some obvious physiological explanations for the morphological differences. For example, the marine samples that were morphologically most similar to the estuarine samples were found in protected areas on the Victorian coastline. The morphological similarity between these samples was due to the size attributes (length, diameter, surface area and volume) and both groups have comparatively large vesicles. Estuarine samples, which are protected from wave and wind action would live in similar environmental conditions to sheltered marine samples, and this may influence vesicle size. Chains of large vesicles may be more easily torn apart by waves and accordingly large vesicles are found in sheltered areas. Bergquist (1959) suggested that the physiological reason for larger vesicles in estuarine thalli was the lack of exposure to wave action. However. Ralph et al. (1998) noted that there is no experimental evidence to suggest that wave action is the determining factor rather than other conditions such as salinity or desiccation. The current evidence provides support for Bergquist's (1959) suggestion, since it was the sheltered marine samples which were most similar to the estuarine samples. Furthermore, several authors have highlighted the importance of exposure to wave action as a morphological determinant (Bhattacharya and Druehl 1989, Miller et al. 2000).

Within the marine dataset, the five distinct geographical groups were distinguished by axis 2 (dominated by shape) of the PCA. These five groups roughly follow Knox's (1963) marine biogeographic provinces of Australia. The southern and northern New South Wales groups fall into the Peronian Province, the Victorian and Tasmanian groups fall into the Maugean Province and the South Australian group lies in the Flindersian Province. The fact that these groups are based on geographical location suggests that they are either influenced by broad-scale environmental differences (such as water temperature) or they have come about due to restricted gamete dispersal, which has led to reduced mixing of genetic material. 

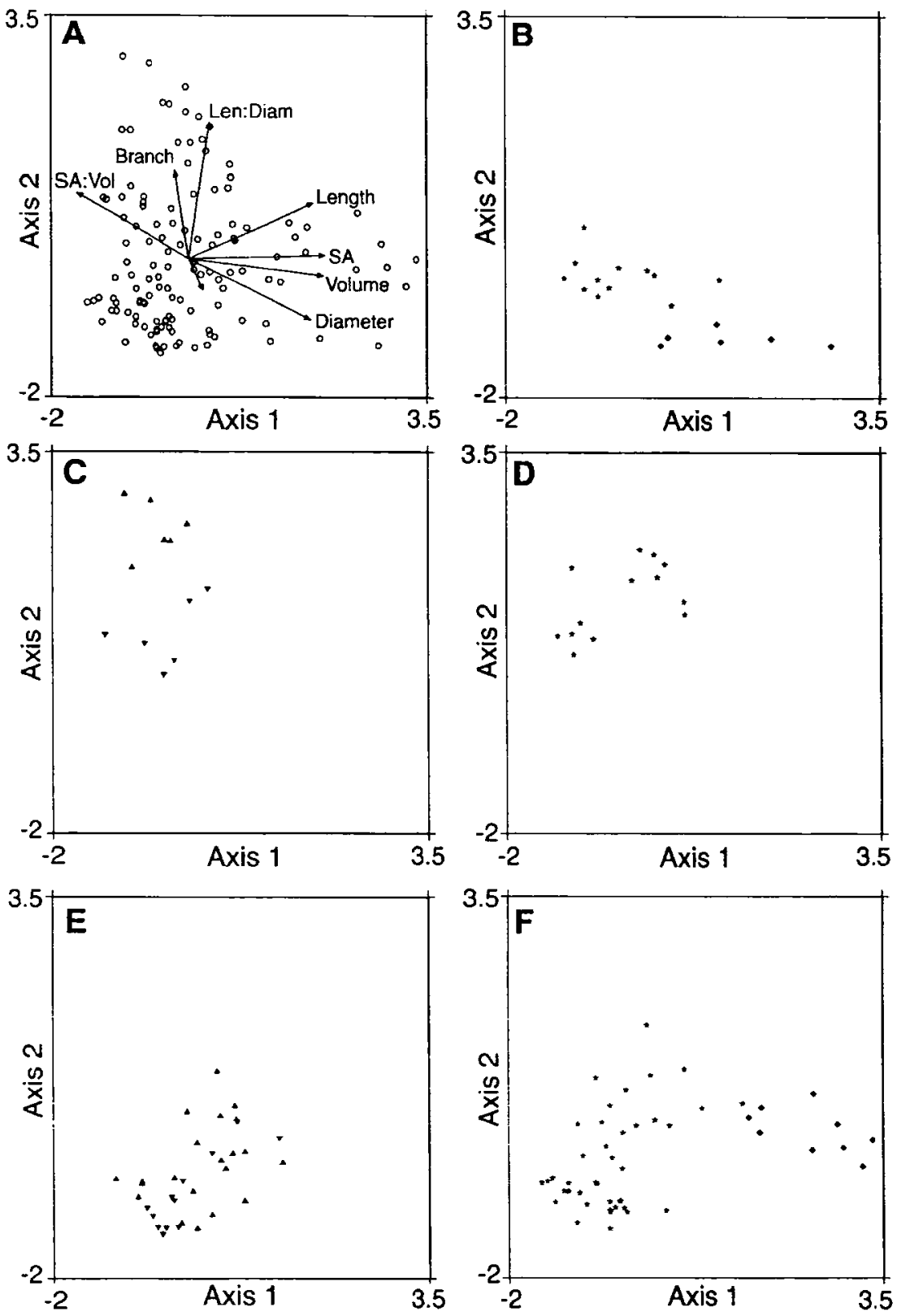

Figure 3 Hormosira banksii: projection of the estuarine individuals onto axes representing the first two functions of the principal components analysis of the vesicle characteristics.

Showing (A) all 125 individuals from all 24 of the sites, along with the variation in each morphological attribute, (B) only those 18 individuals from the tidal flats of the southern coastline ( $\star$ : York Peninsula; $\$$ : Eyre Peninsula), (C, D) only those 25 individuals from the tidal flats of the eastern coastline ( $\mathbf{\Lambda}$ : lower tidal; $\star$ : mid tidal; $\mathbf{~ : ~ u p p e r ~ t i d a l ) , ~ a n d ~}(E, F)$ only those 82 individuals from the mangrove sites ( $\mathbf{\Lambda}$ : lower tidal; $\star$ : mid tidal: $\mathbf{\nabla}$ : upper tidal; $\bullet$ : Salamander Bay site). Each symbol represents one thallus, and the spatial relationship of the symbols represents their relative morphological similarity based on all of the attributes. Each arrow represents one morphological attribute (using the codes indicated in Figure 2; the unlabelled arrow is Shape), the direction of the arrow indicating the direction of maximum variation in that attribute and the length of the arrow indicating the relative amount of morphological change.

The idea of genetic isoloation of marine and estuarine populations is supported by consideration of the reproductive mechanism of Hormosira banksii. This dioecious alga releases gametes when stimulated by warmth and desiccation (Clarke 1978, Gunthorpe et al. 1997), so the release of gametes is more likely while the tide is out. Incoming tidal waters sometimes wash the gametes further up the shore, or eggs may sink to the bottom close to the parent thalli (Bellgrove et al. 2004). A mucilage excretion provides adhesion to the substratum within three hours of fertilisation (Forbes and Hallam 1979) so gametes are rare in the water column (Bellgrove et al. 2004). In this way, progeny are distributed close to their parent thalli, and distinct forms of $H$. banksii may become genetically discrete, a common feature of Phaeophyceae (Johnson and Brawley 1998). Indeed, low concentrations 

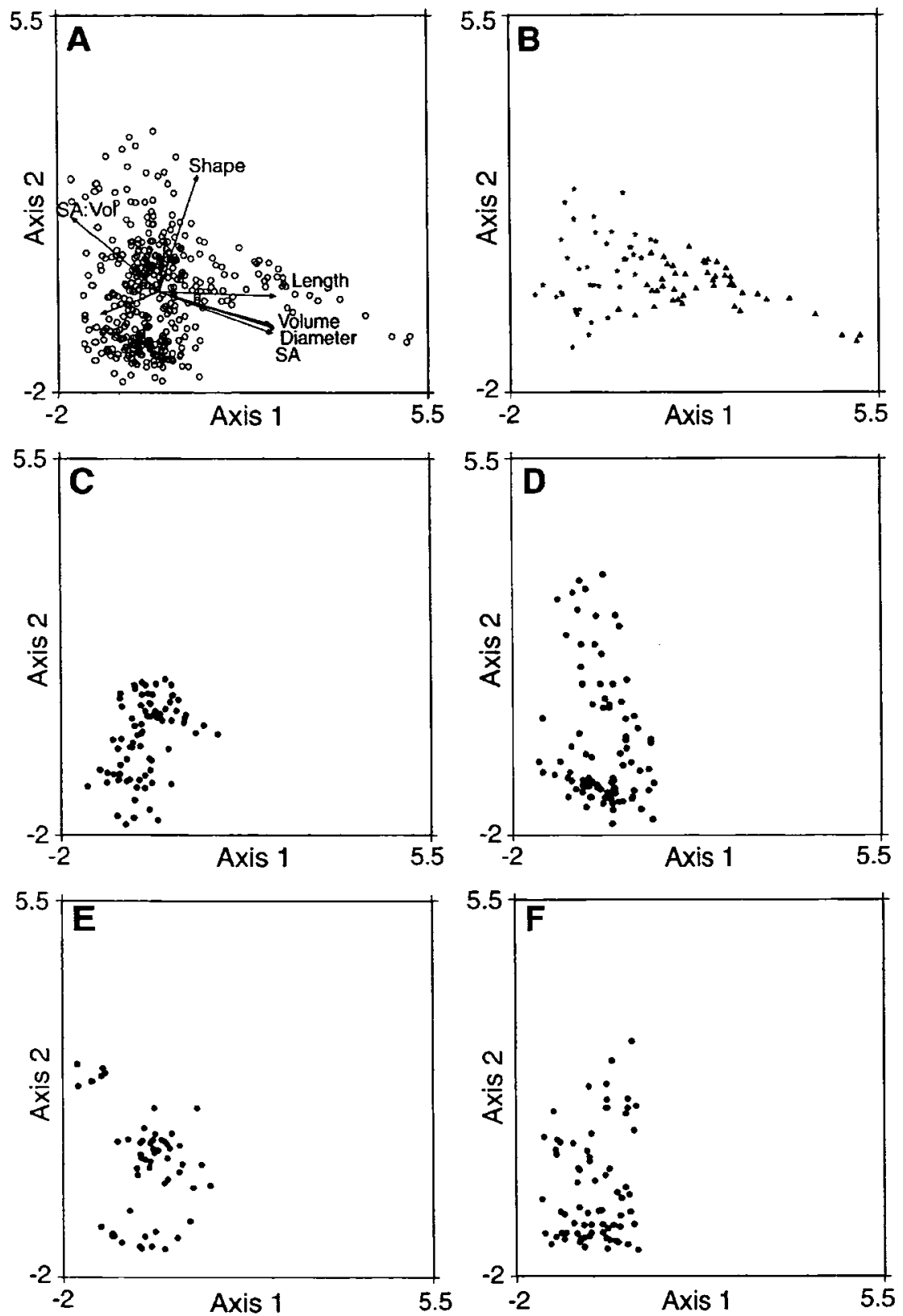

Figure 4 Hormosira banksii: projection of the marine individuals onto axes representing the first two functions of the principal components analysis of the vesicle characteristics.

Showing (A) all 380 individuals from all 50 of the sites, along with the variation in each morphological attribute, (B) only those 84 individuals from the Victorian coastline ( $\boldsymbol{A}$ : sites $26,30,33,34,36,38$; : remaining sites), (C) only those 78 individuals from the northern part of the New South Wales coastline, (D) only those 90 individuals from the southern part of the New South Wales coastline, and $(E)$ only those 57 individuals from the South Australian coastline, and $(F)$ only those 71 individuals from the Tasmanian coastline. Each symbol represents one thallus, and the spatial relationship of the symbols represents their relative morphological similarity based on all of the attributes. Each arrow represents one morphological attribute (using the codes indicated in Figure 2; the unlabelled arrows are Branch and Len:Diam), the direction of the arrow indicating the direction of maximum variation in that attribute and the length of the arrow indicating the relative amount of morphological change.

of $H$. banksii zygotes in water samples from different habitats led Bellgrove et al. (1997) to conclude that this species has limited dispersability and that re-establishment of polluted sites will therefore be very slow, since movement of zygotes is so restricted. Restricted gene flow may also have resulted in the formation of the main groups within the estuarine data set, but this needs to be confirmed with genetic studies.
Different morphological forms were certainly present throughout the range of Hormosira banksii assessed in the current study, but the ecoforms identified by Ralph et al. (1998) do not extend far beyond the range of their restricted sample area. It is unlikely that different attributes measured in the two studies (vesicle wall thickness and therefore cavity volume were not included in the current study while length:breadth ratio and branching 
pattern were) would have a significant impact on the outcome. Yet, including samples from the southern coast would certainly increase the variability and range of environmental conditions, and increase morphological variability concomitantly.

Further studies are required to determine the nature of the response to environment. Determining whether genetic or environmental variation is the most important factor in the expression of morphologies is difficult due to the fact that the intertidal habitat is a constantly changing environment. Diurnal changes come with the tidal cycle, but more importantly, seasonal changes can bring erratic weather conditions which cause desiccation during summer and violent swells during winter. Transplant experiments would be one of the best ways to determine the effect of a particular environment on Hormosira banksii, yet they have been largely unsuccessful in the past (Clarke 1978, King 1981, Macinnis- $\mathrm{Ng}$ et al. unpublished) although they have the capacity to produce useful results in other species [for example, Serisawa et al. (2003)].

In this regard, it is important to note the considerable temporal variation in morphology that we observed between the 1994 and 1999 samples taken at the same marine (but not estuarine) sites. There was no consistent pattern to this variation, and so it cannot confound any study of the geographical and habitat variation. However, some of this temporal variation may well be a response to variable environmental conditions, and if this is so then it needs to be taken into account in any future studies.

The nature of variation in Hormosira banksii thus appears to be similar to that of Fucus distichus $\mathrm{L}$. (Fucales. Phaeophyta) where the species is composed of a mosaic of significantly different local populations (Rice and Chapman 1985). The taxonomic implications of the current assessment of $H$. banksii morphology remain uncertain. particularly because the species concept for marine algae continues to be debated (Russell 1986). As suggested by Ralph et al. (1998), if the current evidence were taken to demonstrate the presence of different species. the estuarine form would be designated Hormosira banksii (Turner) Decaisne and the marine individuals would be Hormosira seiberi (Bory) Decaisne, following Womersley (1967). We do not believe the current evidence is sufficiently strong to warrant a taxonomic change to $H$. banksii without genetic analysis of the two groups.

\section{Acknowledgements}

We thank A. Fairhead for assistance with sample location in Adelaide and S. Stenlund for assistance with data collection in Tasmania and South Australia. Funding for the field trips was provided by a UTS institutional grant. We thank A. Bellgrove and an anonymous reviewer for comments, which greatly improved the manuscript.

\section{References}

Bellgrove, A., M. Clayton and G. Quinn. 1997. Effects of secondarily treated sewage effluent in intertidal macroalgal recruitment processes. Mar. Freshw. Res. 48: 137-146.
Bellgrove, A., M. Clayton and G. Quinn. 2004. An integrated study of the temporal and spatial variation in the supply of propagules, recruitment and assemblages of intertidal macroalgae on a wave-exposed rocky coast, Victoria, Australia. J. Exp. Mar. Biol. Ecol. 310: 207-225.

Bergquist. P. 1959. A statistical approach to the ecology of Hormosira banksii. Bot. Mar. 1: 23-52.

Bhattacharya, D. and L. Druehl. 1989. Morphological and DNA sequence variation in the kelp Costaria costata (Phaeophyta). Mar. Biol. 102: 15-23.

Brawley, S., L. Johnson, G. Pearson, V. Speransky, R. Li and E. Serrao. 1999. Gamete release at low tide in fucoid algae: maladaptive or advantageous? Am. Zool. 39: 218-229.

Burrows, E. and S. Lodge. 1951. Autecology and the species problem in Fucus. J. Mar. Biol. Assoc. UK. 30: 161-175.

Cheshire, A. and J. Conran. 1995. A cladistic analysis of the evolution and biogeography of Durvillaea (Phaeophyta). J. Phycol. 31: 644-655.

Cheshire. A. and N. Hallam. 1989. Morphological differences in the southern bull-kelp (Durvillaea potatorum) throughout South-eastern Australia. Bot. Mar. 32: 191-197.

Clarke. S. 1978. A study of Hormosira banksii (Phaeophyta): the taxonomy, genology, growth and phenology of four morphologically distinct forms on South Australian coasts. Honours Thesis, Department of Botany, The University of Adelaide. pp. 88.

Clarke, S. and H. Womersley. 1981. Cross-fertilisation and hybrid development of forms of the brown alga Hormosira banksii (Turner) Decnaisne. Aust. J. Bot. 29: 497-505.

Clayton. M. 1984. Evolution of the Phaeophyta with particular reference to the Fucales. Prog. Phycol. Res. 3: 11-46.

Coyer, J.. J. Olsen and W. Stam. 1997. Genetic variability and spatial separation in the sea palm kelp Postelsia palmaetormis (Phaeophyceae) as assessed with M13 fingerprints and RAPDs. J. Phycol. 33: 561-568.

Forbes. M. and N. Hallam. 1979. Embryogenesis and substratum adhesion in the brown alga Hormosira banksii (Turner) Decassne. Br. Phycol. J. 14: 69-81.

Gunthorpe, L., M. Nottage, D. Palmer and R. Wu. 1997. Testing for sublethal toxicity using gametes of Hormosira banksii: protocol. National Pulp Mills Research Program. Technical Report 22. Pulp and Paper Research Advisory Board, Melbourne. pp. 62.

Johnson. L. and S. Brawley. 1998. Dispersal and recruitment of a canopy-forming intertidal alga: the relative roles of propagule availability and post-settlement processes. Oecologia 117: 517-526.

King. R. 1981. The free-living Hormosira banksil (Turner) Decaisne associated with mangroves in temperate eastern Australia. Bot. Mar. 24: 569-576.

King. R. and M. Wheeler. 1985. Composition and geographic distribution of mangrove macroalgal communities in New South Wales. Proc. Linn. Soc. New South Wales 108: 97-117.

Knox, G. 1963. The biogeography and intertidal ecology of the Australasian coasts. Oceanogr. Mar. Biol. Annu. Rev. 1: 341-404.

Millar. A. and G. Kraft. 1994. Catalogue of marine brown algae (Phaeophyta) of New South Wales, including Lord Howe Island, south-west Pacific. Aust. Syst. Bot. 7: 1-46.

Miller. K. J. Olsen and W. Stam. 2000. Genetic divergence correlates with morphological and ecological subdivision in the deep-water elk kelp, Pelagophycus porra (Phaeophyceae). J. Phycol. 36: 862-870.

Neefus, C., B. Allen, H. Baldwin, A. Mathieson, R. Eckert, C. Yarish and M. Miller. 1993. An examination of the population genetics of Laminaria and other brown algae in the Laminariales using starch get electrophoresis. Hydrobiologia 260/ 261: $67-79$.

Nizamuddin, M. 1961. Classification and distribution of the Fucales. Bot. Mar. 4: 191-203. 
Osborn, J. 1948. The structure and life history of Hormosira banksii (Turner) Decaisne. Trans. Roy. Soc. New Zealand 77: $47-71$.

Raloh. P., D. Morrison and A. Addison. 1998. A quantitative study of morphological variation within Hormosira banksii (Turner) Decaisne (Phaeophyta: Fucales) in south eastern Australia. J. Exp. Mar. Biol. Ecol. 225: 285-300.

Rice, E.L. and A.R.O. Chapman. 1985. A numerical taxonomic study of Fucus distichus (Phaeophyta). J. Mar. Biol. Assoc. UK. 65: 433-459.

Russell, G. 1978. Brown algae: environment and form. In: (D. Irvine and J. Price, eds) Modern approaches to the taxonomy of red and brown algae. Systematics Association special volume 10. Academic Press, New York. pp. 339-369.

Russell, G. 1986 Variation and natural selection in marine macroalgae. Oceanogr. Mar. Biol. Annu. Rev. 24: 309-377.

Serisawa, Y., M. Aoki, T. Hirata. A. Bellgrove, A. Kurashima, Y. Tsuchiya, T. Sato, H. Ueda and Y. Yokohama. 2003. Growth and survival rates of large-type sporophytes of Ecklonia cava transplanted to a growth environment with small-type sporophytes. J. App. Phycol. 15: 311-318. ter Braak, C. 1988. CANOCO - an extension of DECORANA to analyse species-environment relationships. Vegetatio 75 : $159-160$.

Williams, S. and R. Di Fiori. 1996. Genetic diversity and structure in Pelvetia fastigiata (Phaeophyta: Fucales): does a small effective neighborhood size explain fine-scale genetic structure? Mar. Biol. 126: 371-382.

Womersley, H. 1967. A critical survey of the marine algae of southern Australia. Aust. J. Bot. 35: 189-270.

Womersley, H. 1981. Aspects of the distribution and biology of Australian marine macro-algae. In: (J. Pate and P. McComb, eds) The biology of Australian plants. University of Western Australia Press, Perth. pp. 294-306.

Womersley, H. 1987. The marine benthic flora of southem Australia. Part II. South Australian Government Printer, Adelaide. pp. 484.

Zar, J. 1984. Biostatistical analysis. Prentice-Hall, Englewood Clifts. pp. 718 .

Received 8 April, 2004; accepted 22 April, 2005 